\title{
Density Functional Simulation of a Breaking Nanowire
}

Nakamura, A.; Brandbyge, Mads; Hansen, Lars Bruno; Jacobsen, Karsten Wedel

Published in:

Physical Review Letters

Link to article, DOI:

10.1103/PhysRevLett.82.1538

Publication date:

1999

Document Version

Publisher's PDF, also known as Version of record

Link back to DTU Orbit

Citation (APA):

Nakamura, A., Brandbyge, M., Hansen, L. B., \& Jacobsen, K. W. (1999). Density Functional Simulation of a Breaking Nanowire. Physical Review Letters, 82(7), 1538-1541. https://doi.org/10.1103/PhysRevLett.82.1538

\section{General rights}

Copyright and moral rights for the publications made accessible in the public portal are retained by the authors and/or other copyright owners and it is a condition of accessing publications that users recognise and abide by the legal requirements associated with these rights.

- Users may download and print one copy of any publication from the public portal for the purpose of private study or research.

- You may not further distribute the material or use it for any profit-making activity or commercial gain

- You may freely distribute the URL identifying the publication in the public portal 


\title{
Density Functional Simulation of a Breaking Nanowire
}

\author{
A. Nakamura, * M. Brandbyge, ${ }^{\dagger}$ L. B. Hansen, and K. W. Jacobsen \\ Center for Atomic-scale Materials Physics and Physics Department, Technical University of Denmark, DK 2800 Lyngby, Denmark
}

(Received 20 May 1998)

\begin{abstract}
We study the deformation and breaking of an atomic-sized sodium wire using density functional simulations. The wire deforms through sudden atomic rearrangements and smoother atomic displacements. The conductance of the wire exhibits plateaus at integer values in units of $2 e^{2} / h$ corresponding to a specific number of eigenchannels. The transitions between plateaus can be abrupt in connection with structural rearrangements or extend over a few $\AA$ of elongation. The interplay between conductance modes and structural deformation is discussed by means of the eigenchannel transmission probabilities. [S0031-9007(99)08496-3]
\end{abstract}

PACS numbers: 73.40.Jn, 62.20.Fe, 73.20.Dx, 73.23.Ad

The abilities to produce, manipulate, and study nanometer scale structures have developed immensely over the past years [1]. One way to produce and investigate metallic nanocontacts has been to bring two pieces of metal (for example, a scanning microscope tip and a surface) into contact and separate them again under controlled conditions. Contacts formed in this way can exhibit quantized conductance [2-7] and they may also show interesting mechanical behavior as, for example, indicated by sudden jumps in measured force curves of Au contacts during breaking [8].

The mechanical and transport properties of metallic nanocontacts may be influenced both by the atomic (geometrical) structures of the contacts and by the confinement of the electronic motion, and a number of experimental $[8,9]$ and theoretical $[4,10-13]$ studies have discussed the interplay between the two. The combined scanningtunneling and atomic-force microscopy experiments by Agraï et al., for example, indicate a very close correspondence between sudden changes in the force and in the conductance during contact elongation. However, on the theoretical side, previous calculations of the conductance and force as a function of elongation have been based on non-self-consistent approaches, where the atomic structures of the contacts have either been not considered at all (i.e., jellium models [11]) or have been determined by interatomic potentials which do not include effects from the confinement of the electronic motion $[4,9,10]$. The need for a self-consistent treatment of the electronic and ionic degrees of freedom is also emphasized by recent suggestions [14] that the quantization of the electronic motion in the contacts may directly influence the force response of the contacts.

In this study we present fully self-consistent densityfunctional theory (DFT) calculations of the atomic structures of a Na nanocontact during elongation until the point where the contact finally breaks. The force response of the system is determined as a function of elongation and the conductance of the contact is calculated based on the effective Kohn-Sham one-electron potential [15]. The contact is seen to be plastically deformed through a combination of mechanical instabilities and smooth atomic displacements. The conductance curve exhibits plateaus at some integer values times the conductance quantum, and at the plateaus the conductance can be ascribed to a specific number of eigenchannels contributing to the transmission. We show that the transitions between the plateaus may occur suddenly, associated with an abrupt atomic rearrangement, or can take place gradually over an elongation range of a few $\AA$. The calculated density of states in the contact region is rather smooth, indicating only weak quantization effects on the obtained force in the last part of the breaking process.

The electronic structure, total energy, and forces on the ions are calculated within the local-density approximation with a standard pseudopotential DFT code [16] using a supercell with periodic boundary conditions (PBC). The starting configuration for the simulation is a straight wire with a hcp-type structure, as shown in Fig. 1. The supercell contains six layers with a total of 39 atoms and is constructed by stacking alternating layers containing six and seven $\mathrm{Na}$ atoms. The distance between the centers of neighboring wires is $17.5 \AA$.

The simulation is carried out as follows: The wire is elongated in small steps by expanding the unit cell in the direction along the wire. In each elongation step, the unit cell is dilated uniformly by $0.4 \AA$ for $16.6 \leq L<$ $19.8 \AA$ and by $0.2 \AA$ for $19.8 \leq L \leq 31.4 \AA$, where $L$ is the height of the unit cell. After each step, all of the atoms are relaxed to a local minimum energy configuration where the forces on the atoms vanish [17]. This procedure corresponds, in principle, to a slow stretching of the wire at very low temperatures, where all thermal diffusion processes are neglected and where the heat transport away from the contact region is efficient enough to keep the contact cold despite the work done by the applied force.

The structures at different heights of the unit cell are shown in the upper part of Fig. 1. In Fig. 2 the total energy and force are shown as a function of elongation together with the sum of atomic displacements in each step. In agreement with previous simulations $[4,10,12,13,18]$ for 


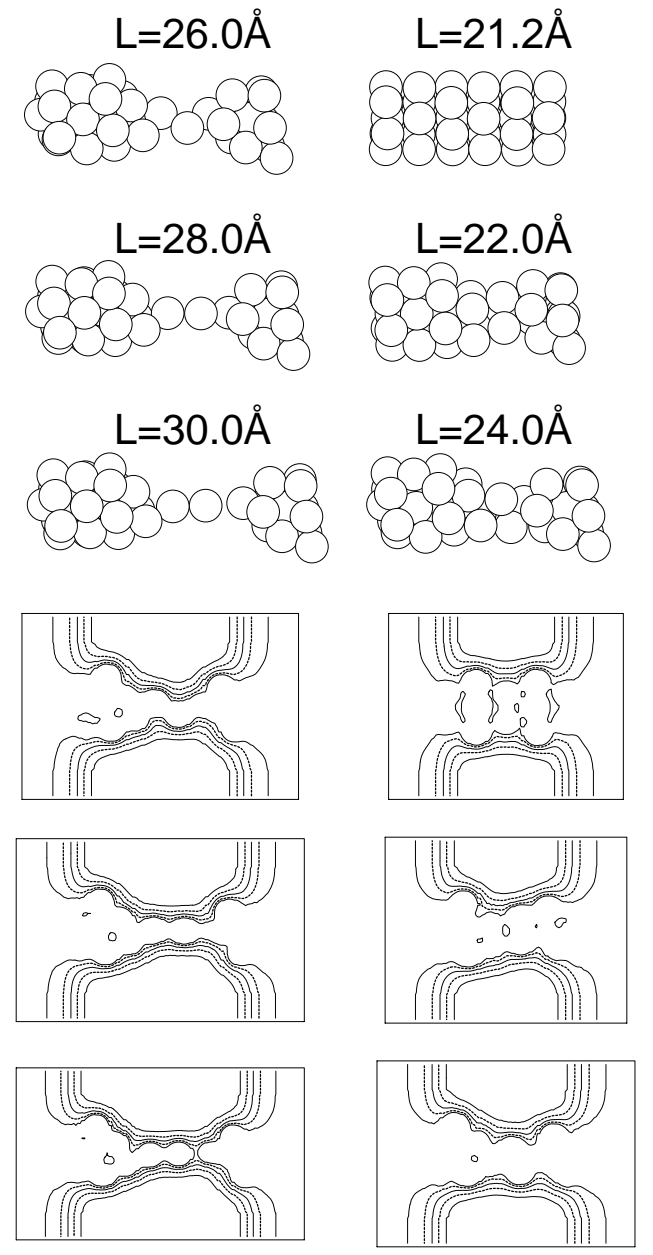

FIG. 1. Calculated atomic structures for different heights, $L$, of the unit cell. Periodic boundary conditions are applied in the calculation. Below, the corresponding cross-sectional contours $\left(E-E_{F}=-3,-2, \ldots,+1 \mathrm{eV}\right)$ of the potentials constructed for the transmission calculation.

various metals, the breaking is seen to involve a series of structural rearrangements which show up as discontinuities in the total-energy and force curves. In the first part of the simulation the wire is elastically stretched and the force builds up until a major atomic rearrangement occurs in which an additional layer of atoms is introduced, as shown in the upper part of Fig. 1 (see $L=21.2 \AA$ and $22.0 \AA$ ). The remaining part of the breaking process also involves some rearrangements but the force never really builds up between the rearrangements. This lack of force buildup for the $\mathrm{Na}$ wire is in contrast to the case of $\mathrm{Au}$, where experiments [8] and simulations [4] display force buildup all the way to the breaking point.

We evaluate the conductance through the wire for each of the obtained relaxed atomic configurations by attaching the wire to free-electron leads and calculating the transmission probability through the formed contact. In this procedure we connect the potential calculated self-consistently within the unit cell $\left[v_{\text {u.c. }}(\vec{r})\right]$ to free-electron electrodes in the following way: Let the $z$ axis be along the direction of

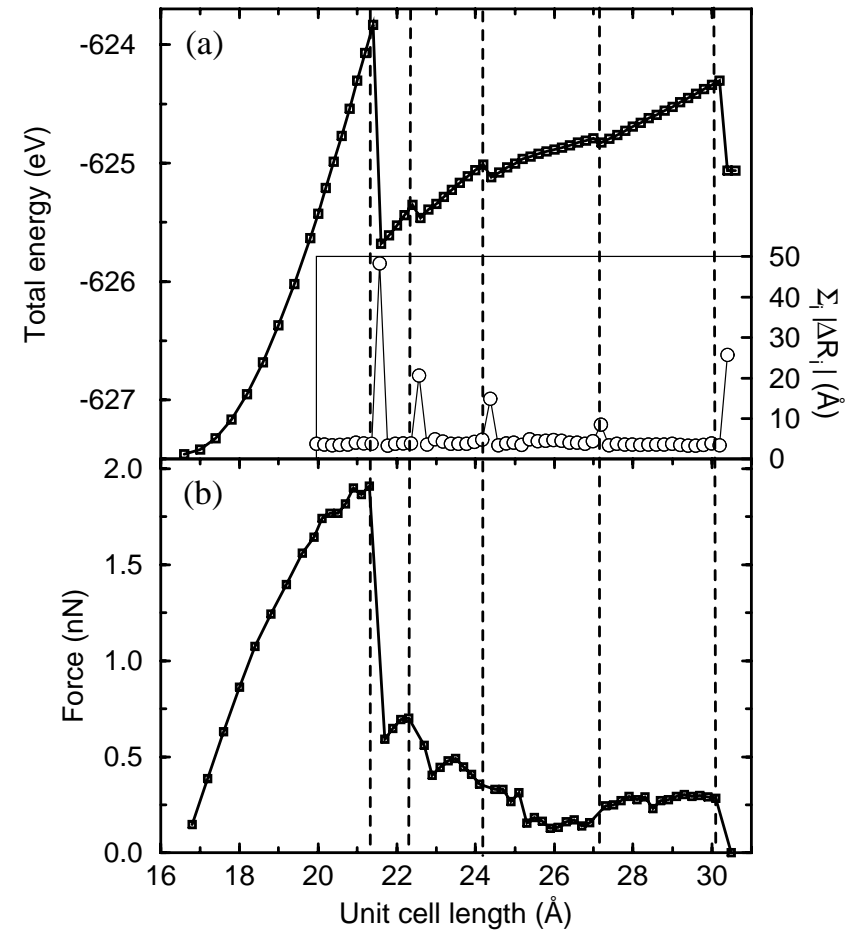

FIG. 2. The total energy of the unit cell, the sum of atomic displacements in each step (a), and the force (b) as a function of the height of the unit cell. The dashed lines indicate the rearrangement points of the atomic configuration. At these points, the total energy jumps discontinuously. The contact breaks at the last dashed line.

the wire and let the narrowest part of the wire be located close to $z=0$ (which we also take as the center of the unit cell: $\left.z_{1} \leq z \leq z_{2}\right)$. The total potential $V(\vec{r})$ is then constructed as

$$
V(\vec{r})=f\left(z_{1}-z\right)\left[v_{\text {u.c. }}(\vec{r})+E_{F}\right] f\left(z-z_{2}\right)-E_{F},
$$

where we use a smoothing function $f(x)=1 /\{1+\exp \times$ $(x / s)\}(s=1 \AA)$. The constructed potential goes to $-E_{F}$ (the electrode bandbottom) inside the electrodes, and in the vacuum it goes to the calculated maximum value of the potential at the sides of the unit cell.

We note that the narrowest region of the potential is by far the most important part regarding the transmission [19], and we emphasize that our results do not depend significantly on the detailed choice of connection as long as it is sufficiently smooth.

We calculate the conductance from the transmission matrix, $\mathbf{t}\left(E_{F}\right)$, using the Landauer-Büttiker formula [20]

$$
G=G_{0} \operatorname{Tr}\left[\mathbf{t}^{\dagger} \mathbf{t}\right],
$$

where $\mathbf{t}$ is obtained using a recursive multichannel method [21].

Recently, conductance eigenchannels have been introduced in the context of nanowires in order to understand the underlying channels for conduction [13]. Each of the 
eigenchannels (index " $n$ ") can be ascribed a transmission probability, $\left|\tau_{n}\right|^{2}$, between 0 and 1 , and the total conductance can be viewed as consisting of independent eigenchannel contributions:

$$
G=G_{0} \sum_{n}\left|\tau_{n}\right|^{2}
$$

In Fig. 3 we show the conductance and its eigenchannel partition of each relaxed atomic configuration during the elongation. We find that the number of open conductance channels roughly corresponds to the number of bands in the band structure of the PBC calculation which crosses or approaches $E_{F}$.

Before the first rearrangement, we observe a smooth decrease of the conductance from $6 G_{0}$ to just above $4 G_{0}$ as the wire cross section smoothly narrows in. We note here that, in this particular stage, the wire is straight and homogeneous (inside the DFT unit cell). Therefore, the details of the scattering at the places where the electrodes are connected to the wire become more important.

After the first rearrangement, a bottleneck containing three atoms has appeared (see $L=22.0 \AA$ in Fig. 1) corresponding to a conductance just below $3 G_{0}$. Inspecting the potential around the bottleneck reveals that it is quite rotational symmetric [12]. This leads to near-degeneracy of two eigenchannels which is reflected in the fact that the conductance moves from $3 G_{0}$ to $G_{0}$ by the almost simultaneous closing of two channels, as shown in Fig. 3.

The first and last restructuring events lead to abrupt changes in the conductance. However, at the three smaller restructurings occurring in between (Fig. 2), the change in conductance is much less dramatic.

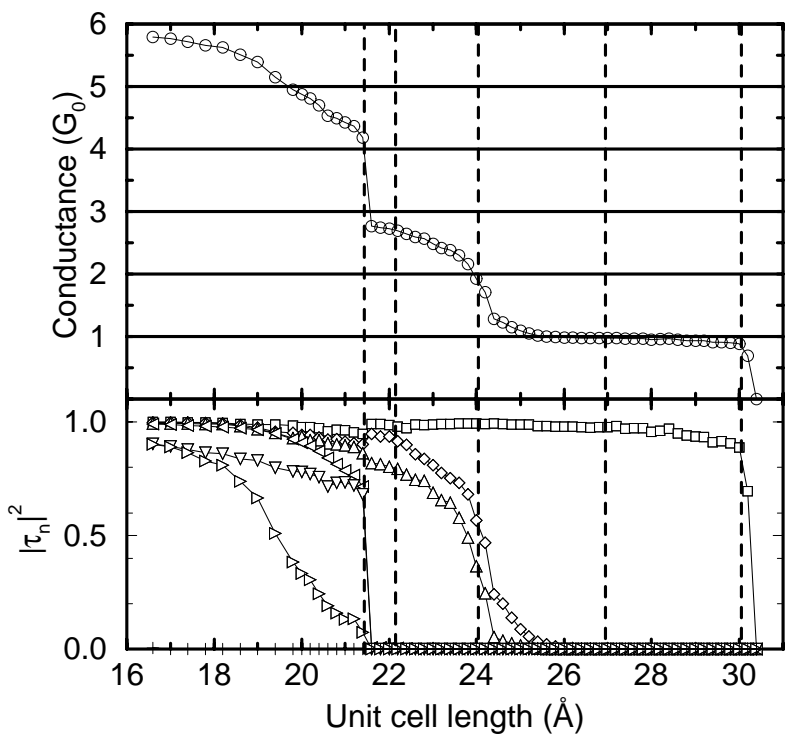

FIG. 3. Conductance calculated using the self-consistent local-density approximation potential of the relaxed atomic configurations during the elongation. Below, we show the eigenchannel transmission probabilities, $\left|\tau_{n}\right|^{2}$, which sum up to the total transmission.
The calculated conductance curve can be compared with the experimental data for $\mathrm{Na}$ contacts at low temperatures $[1,5]$. It can be noted that the simultaneous closing of the second and third eigenchannels corresponds nicely with the observed absence of a peak at $2 G_{0}$ in conductance histograms obtained from breaking many contacts. The experimental conductance curves typically involve sudden jumps, but occasionally also gradual changes can be observed. In fact, one of the curves in Ref. [1] (Fig. 3) shows a gradual change of the conductance from $3 G_{0}$ to $G_{0}$ over 1-2 $\AA$, much like what is seen in this simulation. The gradual change has been interpreted as due to a bistability of the system [1], but in the simulation the phenomenon occurs because of a rather smooth closing of two conduction channels.

We now address the question about the interplay between the electronic states and the structural changes. It was recently suggested [14] that the quantization of the electronic motion in a wire could significantly affect the mechanical force. This effect may arise if the density of states (DOS) shows pronounced peaks and the peaks move through the Fermi level in connection with structural changes. In the upper panel of Fig. 4 we show the DOS inside the unit cell in the PBC calculation (a small broadening of the levels of $0.1 \mathrm{eV}$ is used). In the initial straightwire configuration $(L=21.2 \AA)$ very pronounced peaks are observed at the onset of the different bands. The peaks decay roughly as $1 / \sqrt{E}$ on the high energy side as for ideal

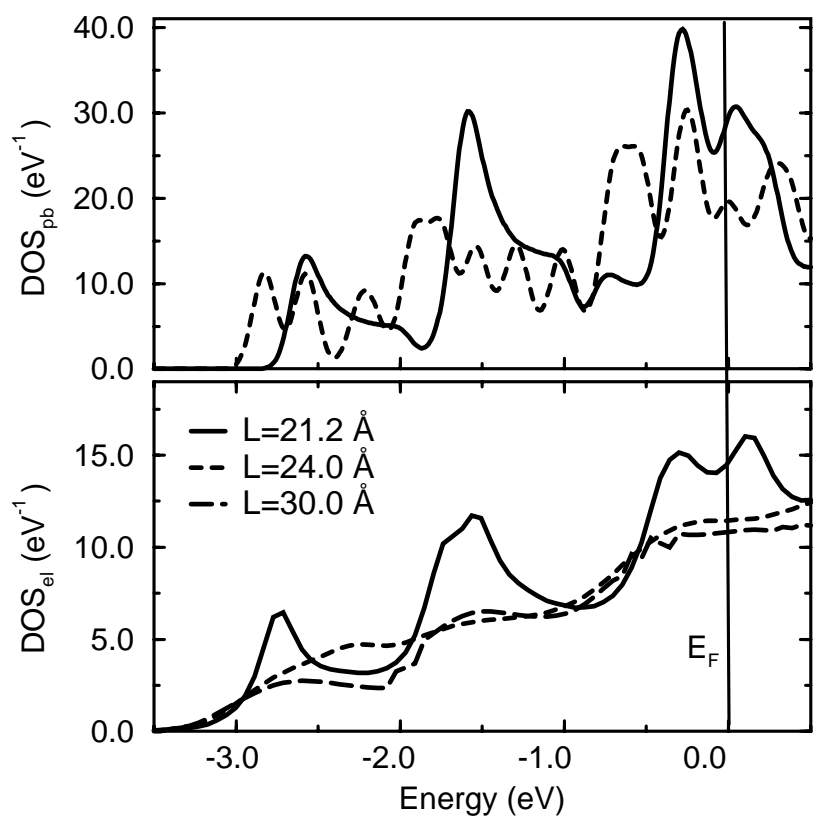

FIG. 4. Upper: Density of states (DOS) inside the unit cell in the self-consistent calculation for $L=21.2 \AA$ (solid line) and $L=24.0 \AA$ (dotted line). For clarity we do not show the very peaked DOS for $L=30.0 \AA$. Lower: Local DOS (integrated over the part of the wire in between the electrodes) for the nanowire coupled to the electrodes. The DOS is seen to be quite smooth after the first restructuring. 
1D free-electron bands. After the first structural rearrangement $(L=24.0 \AA)$, the peaks get much less pronounced; however, a series of smaller and more closely spaced new peaks are seen to develop. These are mostly caused by the PBC: In the limit where the contact breaks, the system consists of a row of independent "quantum dots" with a discrete set of energy levels. In the lower panel of Fig. 4 we show the local DOS (LDOS) integrated over the wire part [22] in the case where free-electron leads are attached. For the ideal initial wire configuration $(L=21.2 \AA)$ we again see clear peaks in the LDOS curve corresponding to the onset of the bands and a reasonable agreement on the peak locations with the upper panel. However, after the first restructuring the LDOS becomes much smoother. The series of smaller closely spaced peaks seen in the PBC case is completely smeared out due to the coupling to the electrodes. The calculations therefore seem to indicate that the peaked nature of the density of states seen for the idealized homogeneous wire structures can be suppressed for more realistic contact geometries.

In conclusion, we studied the interplay between the conductance modes and the structural changes in Na nanocontacts during elongation with the use of DFT simulations. The conductance exhibits plateaus at integer value times $2 e^{2} / h$, and the transitions between plateaus can be either abrupt in connection with structural rearrangements or extend over a few $\AA$ of elongation.

We gratefully acknowledge discussions with J.K. Nørskov and B. Hammer. A. N. expresses sincere thanks to A. Okiji. CAMP is sponsored by the Danish National Research Foundation.

*Permanent address: Dept. of Physics, Anan College of Technology, Anan, Tokushima 774, Japan.

†Present address: MIC, Technical University of Denmark (DTU), Build. 345 east, DK-2800, Denmark.

[1] For a recent review, see, e.g., J.M. van Ruitenbeek, in Mesoscopic Electron Transport, edited by L. L. Sohn et al. (Kluwer, Netherlands, 1997), pp. 549-579.

[2] N. Agraït, J. G. Rodrigo, and S. Vieira, Phys. Rev. B 47, 12345 (1993).

[3] L. Olesen et al., Phys. Rev. Lett. 72, 2251 (1994).

[4] M. Brandbyge et al., Phys. Rev. B 52, 8499 (1995).

[5] J. M. Krans et al., Nature (London) 375, 767 (1995).

[6] J. L. Costa-Krämer, N. García, and H. Olin, Phys. Rev. B 55, 12910 (1997).

[7] T. Junno et al., Appl. Phys. Lett. 72, 548 (1998).

[8] G. Rubio, N. Agraï, and S. Vieira, Phys. Rev. Lett. 76, 2302 (1996).

[9] U. Landman, W. D. Luedtke, B. E. Salisbury, and R. L. Whetten, Phys. Rev. Lett. 77, 1362 (1996).
[10] T. N. Todorov and A. P. Sutton, Phys. Rev. Lett. 70, 2138 (1993).

[11] J. A. Torres and J. J. Sáenz, Phys. Rev. Lett. 77, 2245 (1996).

[12] R. N. Barnett and U. Landman, Nature (London) 387, 788 (1997).

[13] M. Brandbyge, M. R. Sørensen, and K. W. Jacobsen, Phys. Rev. B 56, 14956 (1997).

[14] C. A. Stafford, D. Baeriswyl, and J. Bürki, Phys. Rev. Lett. 79, 2863 (1997).

[15] W. Kohn and L. J. Sham, Phys. Rev. 140, A1133 (1965).

[16] B. Hammer and O. H. Nielsen in Applied Parallel Computing, edited by J. Dongarra et al. (Springer, Berlin, 1996); G. Kresse and J. Furthmüller, Comput. Mater. Sci. 6, 15 (1996); N. Troullier and J. L. Martins, Phys. Rev. B 43, 1993 (1991). Exchange correlation: J.P. Perdew and A. Zunger, Phys. Rev. B 23, 5048 (1981) combined with nonlinear core correction [S.G. Louie, S. Froyen, and M. L. Cohen, Phys. Rev. B 26, 1738 (1982)]. We use 8 special $k$-points in the irreducible part of the 1D Brillouin zone. The energy cutoff of the plane-wave basis set is $7 \mathrm{Ry}$. The convergence was checked using cutoffs of 5, 7, and 9 Ry and with 4, 6, 8, and $16 k$-points.

[17] The energy minimization is carried out with the molecular dynamics minimization technique: P. Stoltze, Simulation Methods in Atomic-Scale Materials Physics (Polyteknisk Forlag, Lyngby, 1997). The time step used is $3 \mathrm{fs}$. The energy is minimized until the forces on the atoms become smaller than $0.05 \mathrm{eV} / \AA$. At the start of each relaxation procedure the atoms are randomly displaced with the use of a position probability distribution of a harmonic oscillation, $2 /\left\{r_{0} \pi \cos \left[\sin ^{-1}\left(r / r_{0}\right)\right]\right\}$, to a random direction. The maximum displacement, $r_{0}$, is decided from the temperature. We use $r_{0}=0.1 \AA$, roughly corresponding to equilibrium displacements at $10 \mathrm{~K}$. This stochastic noise is introduced in order to break any symmetries from the previous steps.

[18] U. Landman, W. D. Luedtke, N. A. Burnham, and R. J. Colton, Science 248, 454 (1990).

[19] M. Brandbyge, K. W. Jacobsen, and J. K. Nørskov, Phys. Rev. B 55, 2637 (1997).

[20] M. Büttiker, Y. Imry, R. Landauer, and S. Pinhas, Phys. Rev. B 31, 6207 (1985).

[21] K. Hirose and M. Tsukada, Phys. Rev. B 51, 5278 (1995); Ref. [19], and references therein. A $x y$ unit cell with sides $L_{x}=L_{y}=20 \AA$ and $x y z$ grid spacing of $0.16 \AA$ are used. With this method, we are restricted to using a reasonable small number of plane waves. Therefore we neglect the scattering by the atomic cores. This is done simply by cutting off the wire potential, $v_{\text {u.c. }}(\vec{r})$, at $-E_{F}$. We use $E_{F}=4 \mathrm{eV}$. For this value we obtain approximately the same electron density in the middle of the wire (initial configuration) in the scattering calculation and in the PBC calculation. The transmission and DOS are then converged using 124 channels.

[22] We obtain the LDOS from the $\mathbf{t}(E)$ matrix [M. Brandbyge and M. Tsukada, Phys. Rev. B 57, R15 088 (1998)]. 